Published in final edited form as:

Annu Rev Med. 2019 January 27; 70: 211-224. doi:10.1146/annurev-med-041317-102715.

\title{
Progress in Understanding and Treating Idiopathic Pulmonary Fibrosis
}

\author{
Jonathan A. Kropski ${ }^{1,2}$ and Timothy S. Blackwell ${ }^{1,2,3}$ \\ ${ }^{1}$ Division of Allergy, Pulmonary and Critical Care Medicine, Department of Medicine, Vanderbilt \\ University Medical Center, Nashville, Tennessee 37232, USA; timothy.blackwell@vanderbilt.edu, \\ jon.kropski@vanderbilt.edu 2Department of Veterans Affairs Medical Center, Nashville, \\ Tennessee 37212, USA ${ }^{3}$ Department of Cell and Developmental Biology, Vanderbilt University \\ School of Medicine, Nashville, Tennessee 37212, USA
}

\begin{abstract}
This is a time of substantial progress in the evaluation and care of patients with idiopathic pulmonary fibrosis (IPF). In addition to the approval and widespread availability of the first IPFspecific therapies, there have been improvements in imaging interpretation and lung biopsy methods to enable more expeditious and more accurate diagnosis. Recent advances in identifying genetic factors that underlie susceptibility to IPF and affect prognosis have raised the possibility of personalized therapeutic approaches in the future. Further, evolving work is elucidating novel mechanisms influencing epithelial, mesenchymal, and inflammatory cell responses during the injury-repair process, thus advancing understanding of disease pathogenesis. As analytic approaches mature, the field is now poised to harness the power of rapidly advancing "omics" technologies to further accelerate progress.
\end{abstract}

\section{Keywords}

interstitial lung disease; familial interstitial pneumonia; genetics; alveolar epithelial cell; fibroblast; cryobiopsy

\section{INTRODUCTION}

Interstitial lung diseases (ILDs) represent a heterogeneous group of pulmonary parenchymal disorders resulting from a variety of environmental insults, systemic diseases, and idiopathic conditions. Of the ILDs, idiopathic pulmonary fibrosis (IPF) is the most common and most severe. Current estimates are that $>100,000$ people in the United States and Europe are living with IPF (1). These patients have an average life expectancy of 3-5 years after diagnosis in the absence of lung transplantation. While morbidity and mortality associated with this disease remain unacceptably high, there has been rapid progress in a number of

DISCLOSURE STATEMENT

Dr. Kropski reports grants from Boehringer Ingelheim and study support from Genentech. Dr. Blackwell reports grants from Boehringer Ingelheim and Celgene, and study support from Genentech. 
areas over the last several years, including improved approaches for diagnosis, development of two therapies that have been approved by the US Food and Drug Administration (FDA), and identification of underlying genetic predisposition. The term IPF has been in common use since the 1970s and is linked to the pathological entity of usual interstitial pneumonia (UIP), which is defined by predominant subpleural fibrosis with fibroblastic foci, temporal heterogeneity, and microscopic honeycombing. Understanding of the pathobiology of IPF continues to evolve, with strong evidence supporting the concept that repetitive injury to susceptible alveolar epithelial cells (AECs) drives pathological interactions with fibroblasts, leading to excessive matrix deposition that destroys gas-exchanging units. Progress in understanding and treating IPF has led some investigators to call for renaming this disease to better align with current understanding of disease pathogenesis, as well as its relationship with other ILDs (2). This review focuses on areas of recent progress and highlights areas where more work is needed.

\section{CURRENT APPROACH TO DIAGNOSIS}

Patients with IPF present with chronic exertional dyspnea, cough, bibasilar inspiratory crackles, and sometimes digital clubbing. IPF patients are more commonly male than female, frequently have a tobacco smoking history, and are typically greater than 60 years old. Pulmonary function testing reveals evidence of restriction and reduced diffusing capacity for carbon monoxide. A diagnosis of IPF requires exclusion of pneumoconiosis, hypersensitivity pneumonitis, drug-induced interstitial lung disease, and rheumatologic disease. High-resolution computerized tomography (HRCT) scanning is now the primary diagnostic modality for evaluating patients with suspected IPF.

Although heterogeneity in radiologic appearance, pathology, and patient characteristics can make the diagnosis of IPF challenging, the approach to diagnosis of IPF is becoming more standardized. In 2011, the American Thoracic Society (ATS), the European Respiratory Society (ERS), the Japanese Respiratory Society (JRS), and the Latin American Thoracic Association (ALAT) jointly issued clinical practice guidelines (3). The ATS/ERS/JRS/ALAT guidelines categorized HRCT appearance as (a) UIP pattern, (b) possible UIP pattern, or (c) inconsistent with UIP pattern based on defined CT scan criteria. The UIP pattern category was defined as subpleural basal predominance of reticular abnormalities and honeycombing with or without traction bronchiectasis, and the absence of features inconsistent with the UIP diagnosis. The possible UIP pattern category was assigned if honeycombing was absent. Based on high-quality evidence that HRCT appearance can be highly specific for IPF, these guidelines indicated that the presence of a UIP pattern in combination with appropriate history and physical exam was sufficient for IPF diagnosis. Surgical lung biopsy was suggested for cases where IPF was suspected but the HRCT appearance did not show the UIP pattern. Using this approach, only about half of individuals with IPF/UIP have HRCT scans that are read as having a UIP pattern (4), so a large number of patients require additional diagnostic studies.

A recent Fleischner Society white paper (4) proposed a modification of these diagnostic criteria taking into account the clinical probability of IPF, which in increased in those who are older than 60 , are current or former smokers, and have no history of other potential 
causes of fibrosis. Under these recommendations, the HRCT pattern is categorized as definite UIP, probable UIP, pattern indeterminate for UIP, or features most consistent with non-IPF diagnosis. These guidelines suggest that a probable UIP pattern in which honeycombing is absent could still be diagnostic for UIP if the clinical probability of IPF is high. This classification also suggests that some patients who would be identified as inconsistent with UIP pattern under the 2011 guidelines could have a reasonable likelihood of UIP on biopsy; therefore, some patients in the category of pattern indeterminate for UIP could still benefit from biopsy if there is high clinical suspicion.

An increasingly frequent occurrence is the detection of asymptomatic interstitial changes on abdominal or chest $\mathrm{CT}$ imaging obtained for other purposes, as it is now recognized that up to $7 \%$ of former tobacco smokers over 50 years of age have identifiable interstitial abnormalities $(5,6)$. Although these interstitial changes can progress, development of clinical symptoms over five-year intervals appears infrequent in unselected populations (7). These patients present a challenge for clinicians, as there is potential for early diagnosis and treatment but also risk of overdiagnosis of ILD/IPF. Several ongoing cohort studies have been designed to provide more clarity as to which patients with early asymptomatic interstitial changes on CT warrant further evaluation and/or early treatment (NCT03437486, NCT03478553).

The recommended modality for lung biopsy is also evolving. While thoracoscopic biopsy is still the standard approach, in-hospital mortality is in the range of $1.7 \%$ for elective procedures and as high as $16 \%$ for nonelective procedures $(8,9)$. Although traditional transbronchial biopsies have limited utility, transbronchial cryobiopsy is emerging as an alternative to surgical biopsy. This procedure results in substantially larger biopsies than transbronchial forceps, and a successful diagnosis can be made in 70-80\% of cases (10). This procedure, however, is not well standardized and is currently best used at experienced centers. In inexperienced hands, the diagnostic yield may not be sufficient, and the risk of bleeding and pneumothorax can be substantial.

Although the most recent clinical practice guidelines do not recommend serologic evaluation for patients with suspected IPF, emerging evidence suggests there may be a distinct subgroup of patients who meet current IPF criteria but have more prominent features of autoimmunity and a better prognosis (11). Further work will be needed to refine classification of these patients and understand whether distinct mechanisms are responsible for their disease.

Because of the uncertainty inherent in the clinical, radiological, and pathological evaluation of patients with suspected IPF, multidisciplinary conferences with interactions among clinicians, radiologists, and pathologists are increasingly seen as necessary for consistent evaluation of patients with ILD. Several studies have shown that multidisciplinary discussion increases diagnostic confidence and may change the final consensus diagnosis in up to $20 \%$ of cases (12). However, outside of academic referral centers, access to multidisciplinary conferences is limited. One promising approach calls for adopting the "virtual multidisciplinary team" model (13) from cancer care centers in order to rapidly expand access and improve diagnostic standardization. 
Since an accurate diagnosis of IPF has implications for prognosis and treatment, development of specific biomarkers would be beneficial if they could limit the need for surgical biopsy and improve specificity using noninvasive or minimally invasive techniques. To date, individual protein biomarkers in blood or bronchoalveolar lavage have not proven to be sensitive or specific enough to improve the accuracy of diagnosis of IPF. Several investigators have studied transcriptional signatures in peripheral blood cells and shown that these differ between IPF patients and controls (14-16), but the potential utility of the peripheral blood transcriptome in IPF diagnosis is uncertain. A more promising approach may be to utilize transcriptional profiling of samples obtained by transbronchial biopsy (17). Several studies have now used a machine learning approach to develop algorithms with high specificity for UIP/IPF that could prove to be clinically useful for improving diagnostic accuracy and minimizing the need for surgical biopsy $(18,19)$.

\section{CLINICAL ADVANCES AND ONGOING CHALLENGES}

The approach to treatment of ILD has changed dramatically over the last several years. The PANTHER-IPF study published in 2012 showed not only that immune-suppressive therapy is ineffective in IPF but that the combination of prednisone, azathioprine, and $\mathrm{N}$-acetyl cysteine (NAC) is harmful (20). This study was followed by landmark studies in 2014 indicating that two antifibrotic therapies, pirfenidone (21) and nintedinab (22), were effective in reducing the decline in forced vital capacity $(\mathrm{FVC})$ in patients with moderately advanced IPF. These studies led to FDA approval of both drugs. Nintedinab is an intracellular tyrosine kinase inhibitor. Pirfenidone is an antifibrotic molecule whose target(s) remains uncertain; however, this drug has been shown to reduce fibroblast proliferation and differentiation (23). Although each of these treatments has substantial gastrointestinal side effects, it is becoming clear that most patients can tolerate these therapies for an extended period and that continued therapy remains effective at reducing FVC decline $(24,25)$. In addition, new studies have suggested that patients with milder disease (FVC $>90 \%$ predicted) have similar reduction in FVC decline compared to patients with more advanced disease, suggesting that early treatment is warranted (26-28). While some investigators have questioned the relevance of reduced FVC decline as an important surrogate endpoint for clinical efficacy in IPF (28a), pooled analyses of clinical trials with pirfenidone (which has been studied in more patients than nintedanib) suggest that long-term treatment modestly reduces all-cause and IPF-related mortality (29). This finding suggests that FVC decline is a relevant surrogate endpoint and that these drugs may have long-term disease-modifying effects. However, neither drug has been shown to reduce symptoms or improve quality of life for patients with IPF. At present, there is no compelling reason to choose one drug over the other in most patients, and patient preference related to administration and potential side effects may sway the choice of treatment. Available data do not provide clear guidance as to the approach to treating a patient who is progressing on pirfenidone or nintedanib. While in practice many experts recommend switching to the other agent, criteria for defining treatment failure have not yet been established. Further, an obvious question is whether combination therapy might have added benefit. Although this remains to be answered, the INJOURNEY trial has recently shown that combination therapy is feasible and tolerable (30). 
A 2015 update to the clinical practice guidelines for IPF by ATS/ERS/JRS/ILAT (31) gave conditional recommendations for use of both pirfenidone and nintedinab and recommended against use of other therapies tested to date, with the exception of antacid therapy, which has been suggested as an interdiction to reduce the injurious effects of gastric acid aspiration in this disease. Despite some contradictory data (32), use of proton pump inhibitors or histamine-2 receptor antagonists was recommended on the basis of retrospective studies. For patients with end-stage or progressive disease, lung transplantation remains the treatment of choice. Available data suggest that IPF patients who undergo bilateral lung transplantation may have improved survival compared to those who undergo single lung transplantation (33).

Several important questions related to the care of IPF patients remain unanswered. Acute exacerbations remain a major cause of morbidity and mortality among IPF patients, and there has been limited progress towards understanding the causes, mechanisms, and optimal treatment of these life-threatening disease complications (33a). Pulmonary hypertension is common in severe IPF and leads to worse outcomes (34), yet it is uncertain whether treating secondary pulmonary hypertension could be beneficial, and studies to date have been negative. In addition, obstructive sleep apnea and sleep-disordered breathing are vastly overrepresented in the IPF population (35), often in the absence of typical symptoms (36), and some investigators have suggested there may be reason to perform sleep studies on all IPF patients (36a). However, it remains to be seen whether obstructive sleep apnea treatment impacts disease outcome. Exercise training can improve six-minute-walk distance in patients with IPF (37), but whether this intervention has durable effects requires additional study. Another important question is whether pirfenidone and nintedinab, approved for IPF, have efficacy in other forms of ILD. Numerous ongoing trials are addressing this issue.

Although there is a clear need for additional therapeutic options, the results of most recent studies involving new agents have been disappointing. A large randomized placebocontrolled study targeting lysyl oxidase-like 2 (LOXL2), which catalyzes collagen crosslinking, was terminated early for futility (38). A randomized study of CC-chemokine ligand 2 (39), which regulates recruitment of monocyte-derived macrophages, was also stopped early, and a randomized study of an anti-IL-13 monoclonal antibody did not achieve efficacy endpoints (40). However, despite these results, the field of IPF clinical research has been buoyed by the introduction of pirfenidone and nintedinab, and more studies with novel therapeutics are planned or ongoing. Some studies have shown promising early-phase data (41).

An important issue in the field of IPF clinical studies relates to the design of future phase III clinical trials. Should potential therapies be evaluated in direct comparison to current therapies or as add-on therapies to one of the approved agents? This issue is especially relevant since many investigators believe that rational combination therapies targeting both upstream factors in the injury-repair process and fibroblast activity/matrix deposition are the best strategy for achieving maximal efficacy. In addition, given the rapid advancement in understanding of genetic susceptibility to IPF (discussed below), it will likely be important to stratify patients in future studies based on genetic variables. The best example of the potential importance of this approach to date is a re-evaluation of the PANTHER-IPF study 
in which patients were genotyped for common single-nucleotide polymorphisms (SNPs) associated with risk for IPF. These investigators found a significant interaction between response to NAC therapy and a SNP in TOLLIP that correlated with efficacy in this subgroup (42), suggesting that personalized pharmacogenetics approaches could be a productive strategy for testing new therapies.

\section{PROGRESS IN UNDERSTANDING GENETIC PREDISPOSITION TO IDIOPATHIC PULMONARY FIBROSIS}

It is now recognized that IPF is a gene-by-environment disease with a heterogeneous set of susceptibility genes, along with an ill-defined group of environmental risk factors that includes tobacco smoking. Both common SNPs and rare genetic mutations have been linked to development of IPF (Table 1) (43). To assess the role of common genetic variation in IPF, several genome-wide association studies (GWAS) have now been performed (44-46), resulting in identification of SNPs at 17 different loci that associate with development of IPF, most notably in the promoter region of the Mucin 5B gene, MUC5B (47). This SNP (rs35705950), which has now been confirmed in multiple studies, is located adjacent to a FOXA2 binding site in a region of the MUC5B promoter that is differentially methylated in IPF (48). The minor ( $\mathrm{T}$ ) allele is present in $~ 18 \%$ of the Caucasian population, compared to 60-70\% of IPF patients of European ancestry and is associated with increased MUC5B mRNA expression in normal (although not IPF) lungs (47). Although minor allele carriers of rs35705950 have increased risk of developing disease, IPF patients who carry the risk allele appear to have slower disease progression than noncarriers (49). rs35705950 is much rarer among IPF patients of Asian ancestry (49a), underscoring a need for further study of genetic risk for IPF in ethnically diverse populations. Animal studies have suggested that $M U C 5 B$ regulates airway host defense (50); however, the mechanisms by which altered MUC5B expression influences fibrotic remodeling remain uncertain.

Information regarding rare genetic variants has been generated primarily from studies in individuals with the familial form of IPF, known as familial interstitial pneumonia (FIP). Currently identified disease-associated genes fit into either the telomerase pathway [telomerase reverse transcriptase (TERT) $(51,52)$, regulator of telomere elongation helicase (RTEL1) (53-55), telomerase RNA component (TERC) (51, 52), dyskerin (DKC1) (57), telomere interacting factor 2 (TINF2) (59), poly(A)-specific ribonuclease (PARN) $(55,60)]$, nuclear assembly factor 1 (NAF1) (60a) or the surfactant protein pathway [surfactant protein C (SFTPC) (61-63), surfactant protein A2 (SFTPA2) (64), and ATP-binding cassette member A3 $(A B C A 3)(65,66)]$. Our current estimate is that $15-20 \%$ of FIP families share a loss-of-function mutation in one of the telomerase pathway genes, with TERT and RTEL1 being the most common (43). Rare genetic variants in the surfactant protein pathway are much less common in FIP, accounting for no more than 1-2\% of cases. Patients with telomerase pathway rare variants have very short telomeres as measured in white blood cells, more rapid disease progression, and often other manifestations of the short-telomere syndrome, including liver and bone marrow disease $(51,52,67,68)$. 
The degree of similarity in the genetic underpinnings of familial and sporadic IPF has been an unresolved question in the field. The prevalence of the MUC5B SNP minor allele appears to be similar in patients with familial and sporadic IPF (47), suggesting that common genetic variants are shared in both forms of the disease. For rare genetic variants, prior studies have shown that mutations in the surfactant protein pathway are uncommon in sporadic IPF (69). In contrast, recent data indicate that rare variants in the telomerase pathway occur at a relatively high frequency in patients with sporadic IPF. A recent study using whole-exome sequencing data from 262 subjects with sporadic IPF and unaffected controls found that rare variants in TERT, RTEL1, and PARN were overrepresented in sporadic IPF cases (70). We recently reported data from whole-genome sequencing of 1,510 patients with sporadic IPF and demonstrated that rare variants in TERT, RTEL1, TERC, and PARN were present in $\sim 8.5 \%$ of IPF patients, significantly higher than the percentage of control populations (71). In addition, this study identified an interaction between rare variants in TERT and the $M U C 5 B$ promoter SNP. These findings showed that the $M U C 5 B$ risk allele was substantially less common in IPF patients who harbored a TERT rare variant than in IPF patients without a telomerase mutation, thus suggesting that the MUC5B polymorphism and TERT rare variants may be separable, independent risk pathways for development of IPF. The finding that rare genetic variants in telomerase pathway genes occur frequently in sporadic IPF points to a potential role for genetic testing. We recently published recommendations for genetic testing in familial IPF (72), and ongoing discussions regarding the role for genetic testing in sporadic IPF are warranted.

Although increasing knowledge regarding the genetics of ILD has not yet translated to improved treatment approaches, identification of disease-associated genes has enhanced understanding of the pathobiology of IPF. The identification of a mutant form of surfactant protein C that segregated with disease in a large FIP family in 2002 (61) led to the identification of endoplasmic reticulum (ER) stress as a common abnormality in IPF epithelium that likely contributes to disease pathogenesis through regulation of epithelial cell survival and repair after injury (73-75). Likewise, the description of telomerase pathway mutations in FIP in $2007(51,52)$ led to the identification of short telomeres as a common phenotype in both familial and sporadic IPF. Overall, peripheral blood cell telomere length is much shorter in IPF than in other chronic degenerative and inflammatory diseases. Short telomeres in peripheral white blood cells $(<10$ th percentile adjusted for age) are identified in at least a third of patients with familial and sporadic IPF $(67,68)$. Short peripheral blood telomeres are an important biomarker that is independently associated with a worse prognosis (76). Telomere length measured in type II AECs, in contrast to peripheral blood cells, is uniformly reduced in $\operatorname{IPF}(58,67)$ and may not correlate well with peripheral blood telomere length (77). A recent study shows that telomere shortening in the lungs is limited to AECs, and telomeres are shorter in cells from fibrotic areas compared to nonfibrotic areas in the IPF lung (79). Although the interpretation of the studies is not entirely clear, one explanation for these findings could be that peripheral blood telomere length primarily reflects genetic risk, whereas telomere shortening in the alveolar epithelium is a common factor in disease progression. Severe reduction in telomere length is known to result in senescence and cell cycle arrest; however, the exact mechanisms relating telomerase mutations and telomere shortening to fibrotic remodeling in the lung are still being explored. 
Although prior animal model studies were disappointing in explicating telomere-related disease mechanisms, two recent papers have shown that epithelial specific deletion of the shelterin protein telomere repeat binding factor 1 (TRF1) results in spontaneous fibrotic remodeling with evidence of DNA damage and accumulation of senescent lung epithelial cells $(80,81)$.

Despite rapid progress in understanding the genetics of pulmonary fibrosis, much remains unknown. The underlying genetic predisposition is unknown in $80 \%$ of FIP patients, and current evidence suggests that a variety of genes may be involved, making identification of additional disease-associated genes challenging. In addition, the relationship between rare and common genetic variants is incompletely understood; however, an important clue might be that several common polymorphisms in telomere-associated genes, including TERT, $T E R C$, and $O B F C 1$, are overrepresented in IPF (44), suggesting that combinations of common and rare genetic variants could work in concert to regulate disease-associated phenotypes like telomere shortening. Further progress in the genetics of IPF will likely require large, well-phenotyped cohorts with expanded genetic search space (i.e., wholegenome sequencing) to investigate promoter, intronic, and other regulatory regions, coupled with assessment of gene expression data and in vitro functional evaluation of individual genetic variants.

\section{EVOLVING UNDERSTANDING OF IDIOPATHIC PULMONARY FIBROSIS PATHOGENESIS}

In UIP, collagen deposition in the distal lung parenchyma is thought to occur in association with accumulation of activated (myo)fibroblasts in areas subjacent to the epithelial surface, which is composed of hyperplastic type II AECs or epithelium with a bronchiolar appearance. Collapse of remodeled alveoli creates focal areas of fibrosis and stretches the adjacent lung parenchyma, resulting in microhoneycombing and traction bronchiolectasis, which are characteristic of the disorder. In addition to AECs and fibroblasts, inflammatory cells are thought to contribute to the pathogenesis of this disorder by modulating epithelialfibroblast interactions. The predominant hypothesis explaining disease pathogenesis is that repetitive, environment-derived (micro)injuries to susceptible AECs result in increased cell death, impaired re-epithelialization, and pathological interactions with fibroblasts that lead to persistent activation with excessive collagen and matrix production. AEC susceptibility is thought to be related to genetic predisposition, aging, or senescence. This paradigm has been bolstered in recent years by genetic data and preclinical models showing that epithelial injury can drive subsequent fibrosis.

The importance of stress responses modulating injury-repair responses in AECs has been an area of active investigation in IPF, and altered mitochondrial function has emerged as a potential factor in AEC dysfunction. In AECs, expression of the mitochondrial protective factor PTEN-induced putative kinase 1 (PINK1) is reduced by aging and ER stress (82), resulting in accumulation of damaged mitochondria and decreased cell viability, both of which are characteristic of AECs in IPF. Recently, it was shown that activating transcription factor 3 (ATF3) can mediate downregulation of the PINK1 promoter (83), thus identifying a 
mechanism linking these processes. Impairment of mitochondrial bioenergetics potentially leads to a feedback loop with persistent ER stress that facilitates fibrotic remodeling. In this regard, it was recently shown that iodothyronine deiodinase 2 (DIO2), an enzyme that activates thyroid hormone, is increased in lungs of patients with IPF (84). This study also showed that DIO2-deficient mice had more severe fibrosis following bleomycin treatment and that supplementation with active thyroid hormone reduced fibrosis and improved mitochondrial bioenergetics through interactions with peroxisome proliferator-activated receptor $\gamma$ coactivator a (PGC-1a) and PINK1 (84). Together, these studies (82-84) suggest that interventions to improve mitochondrial function in AECs could reduce vulnerability of these cells to injury, thus reversing fibrotic susceptibility.

Recovery from injury of the distal lung requires proliferation and differentiation of AECs to reestablish barrier and gas exchange functions. Identification of subpopulations of AECs that are responsible for regeneration and repair of the injured alveolus is an area of intense interest. Several recent high-profile papers have markedly advanced the field by identifying a progenitor cell niche for AECs that appears to be regulated by Wnt signaling $(85,86)$, along with other pathways (87), and may be critical for AEC self-renewal and restoration of homeostasis after injury.

Pathological persistence and activation of fibroblasts are responsible for the excess collagen and other matrix products in IPF. Although the origin of pathogenic fibroblasts remains a topic of debate, it was recently shown that the transcription factor T-box gene 4 (TBX4) is an important lineage marker for myofibroblasts that participate in lung fibrosis (88). In addition, it is increasingly recognized that extracellular matrix is functionally abnormal in IPF and may itself drive fibroblast activation (89). Increased matrix stiffness has been shown to activate fibroblasts via mechanosensing pathways (90), resulting in a feed-forward mechanism of progressive fibrotic remodeling once architectural distortion reaches a tipping point.

In addition to epithelial cells and fibroblasts, recent progress has focused on elucidating the role of macrophages in mediating lung fibrosis. Recent papers have indicated that production of transforming growth factor- $\beta$ (TGF- $\beta$ ) by alternatively activated macrophages plays an important role in fibroblast activation $(91,92)$. Also, an atypical monocyte population, identified as segregated-nucleus-containing atypical monocytes (SatM) (93), was shown to activate fibroblasts through non-TGF- $\beta$-dependent mechanisms during lung fibrosis, thus indicating that several subpopulations of monocytes/macrophages could be involved in regulation of the fibrotic process.

\section{FUTURE DIRECTIONS}

We have highlighted recent progress in improving IPF diagnosis and treatment, defining the underlying genetic susceptibility, and advancing understanding of disease pathogenesis. This is a time of rapid progress in IPF, and there are a number of new techniques and approaches whose promise is yet to be fully realized. Expression profiling of lung and peripheral blood cells has been helpful in identifying pathways that are dysregulated in IPF; however, new techniques such as single-cell RNA sequencing show promise to elucidate key factors that 
contribute to profibrotic interactions between epithelial $(94,95)$, mesenchymal $(96)$, and immune/inflammatory cells. This approach can be layered onto next-generation sequencing data, epigenetic data, and other "omics" information to elucidate a more complete picture of the pathobiology of IPF. Advances in primary cell and organoid culture have the potential to provide important information about cellular interactions that regulate differentiation and reparative capacity. Together, these approaches hold promise for identifying disease-relevant pathways and cellular phenotypes that can be targeted for therapeutic benefit.

\section{ACKNOWLEDGEMENTS}

This work was supported by the National Institutes of Health K08HL130595 (JAK), P01HL92870 (TSB), the Department of Veterans Affairs (TSB), the Francis Family Foundation (JAK), and the Department of Defense PR160522 (TSB).

\section{LITERATURE CITED}

1. Raghu G, Chen SY, Hou Q, et al. 2016 Incidence and prevalence of idiopathic pulmonary fibrosis in US adults 18-64 years old. Eur. Respir. J 48:179-86 [PubMed: 27126689]

2. Wolters PJ, Blackwell TS, Eickelberg O, et al. 2018 Time for a change: Is idiopathic pulmonary fibrosis still idiopathic and only fibrotic? Lancet Respir. Med 6:154-60 [PubMed: 29413083]

3. Raghu G, Collard HR, Egan JJ, et al. 2011 An official ATS/ERS/JRS/ALAT statement: idiopathic pulmonary fibrosis: evidence-based guidelines for diagnosis and management. Am. J. Respir. Crit. Care Med 183:788-824 [PubMed: 21471066]

4. Lynch DA, Sverzellati N, Travis WD, et al. 2018 Diagnostic criteria for idiopathic pulmonary fibrosis: a Fleischner Society white paper. Lancet Respir. Med 6:138-53 [PubMed: 29154106]

5. Hunninghake GM, Hatabu H, Okajima Y, et al. 2013 MUC5B promoter polymorphism and interstitial lung abnormalities. N. Engl. J. Med 368:2192-200 [PubMed: 23692170]

6. Putman RK, Hatabu H, Araki T, et al. 2016 Association between interstitial lung abnormalities and all-cause mortality. JAMA 315:672-81 [PubMed: 26881370]

7. Araki T, Putman RK, Hatabu H, et al. 2016 Development and progression of interstitial lung abnormalities in the Framingham Heart Study. Am. J. Respir. Crit. Care Med 194(12):1514-22 [PubMed: 27314401]

8. Hutchinson JP, McKeever TM, Fogarty AW, et al. 2016 Surgical lung biopsy for the diagnosis of interstitial lung disease in England: 1997-2008. Eur. Respir. J 48:1453-61 [PubMed: 27660509]

9. Durheim MT, Kim S, Gulack BC, et al. 2017 Mortality and respiratory failure after thoracoscopic lung biopsy for interstitial lung disease. Ann. Thorac. Surg 104:465-70 [PubMed: 28527960]

10. Lentz RJ, Argento AC, Colby TV, et al. 2017 Transbronchial cryobiopsy for diffuse parenchymal lung disease: a state-of-the-art review of procedural techniques, current evidence, and future challenges. J. Thorac. Dis 9:2186-203 [PubMed: 28840020]

11. Fischer A, Antoniou KM, Brown KK, et al. 2015 An official European Respiratory Society/ American Thoracic Society research statement: interstitial pneumonia with autoimmune features. Eur. Respir. J 46:976-87 [PubMed: 26160873]

12. Castillo D, Walsh S, Hansell DM, et al. 2018 Validation of multidisciplinary diagnosis in IPF. Lancet Respir. Med 6:88-89 [PubMed: 29413085]

13. Munro AJ, Swartzman S. 2013 What is a virtual multidisciplinary team (vMDT)? Br. J. Cancer 108:2433-41 [PubMed: 23756866]

14. Herazo-Maya JD, Noth I, Duncan SR, et al. 2013 Peripheral blood mononuclear cell gene expression profiles predict poor outcome in idiopathic pulmonary fibrosis. Sci. Transl. Med 5:205ra136

15. Yang IV, Luna LG, Cotter J, et al. 2012 The peripheral blood transcriptome identifies the presence and extent of disease in idiopathic pulmonary fibrosis. PLOS ONE 7:e37708 [PubMed: 22761659] 
16. Herazo-Maya JD, Sun J, Molyneaux PL, et al. 2017 Validation of a 52-gene risk profile for outcome prediction in patients with idiopathic pulmonary fibrosis: an international, multicentre, cohort study. Lancet Respir. Med 5:857-68 [PubMed: 28942086]

17. Pankratz DG, Choi Y, Imtiaz U, et al. 2017 Usual interstitial pneumonia can be detected in transbronchial biopsies using machine learning. Ann. Am. Thorac. Soc 14:1646-54 [PubMed: 28640655]

18. Choi Y, Lu J, Hu Z, et al. 2017 Analytical performance of Envisia: a genomic classifier for usual interstitial pneumonia. BMC Pulm. Med 17:141 [PubMed: 29149880]

19. Kim SY, Diggans J, Pankratz D, et al. 2015 Classification of usual interstitial pneumonia in patients with interstitial lung disease: assessment of a machine learning approach using highdimensional transcriptional data. Lancet Respir. Med 3:473-82 [PubMed: 26003389]

20. Raghu G, Anstrom KJ, King TE, Jr., et al. 2012 Prednisone, azathioprine, and N-acetylcysteine for pulmonary fibrosis. N. Engl. J. Med 366:1968-77 [PubMed: 22607134]

21. King TE, Jr., Bradford WZ, Castro-Bernardini S, et al. 2014 A phase 3 trial of pirfenidone in patients with idiopathic pulmonary fibrosis. N. Engl. J. Med 370:2083-92 [PubMed: 24836312]

22. Richeldi L, du Bois RM, Raghu G, et al. 2014 Efficacy and safety of nintedanib in idiopathic pulmonary fibrosis. N. Engl. J. Med 370:2071-82 [PubMed: 24836310]

23. Selvaggio AS, Noble PW. 2016 Pirfenidone initiates a new era in the treatment of idiopathic pulmonary fibrosis. Annu. Rev. Med 67:487-95 [PubMed: 26565677]

24. Costabel U, Albera C, Lancaster LH, et al. 2017 An open-label study of the long-term safety of pirfenidone in patients with idiopathic pulmonary fibrosis (RECAP). Respir. Int. Rev. Thorac. Dis 94:408-15

25. Richeldi L, Kreuter M, Selman M, et al. 2018 Long-term treatment of patients with idiopathic pulmonary fibrosis with nintedanib: results from the TOMORROW trial and its open-label extension. Thorax 73:581-83 [PubMed: 28993537]

26. Albera C, Costabel U, Fagan EA, et al. 2016 Efficacy of pirfenidone in patients with idiopathic pulmonary fibrosis with more preserved lung function. Eur. Respir. J 48:843-51 [PubMed: 27471208]

27. Costabel U, Inoue Y, Richeldi L, et al. 2016 Efficacy of nintedanib in idiopathic pulmonary fibrosis across prespecified subgroups in INPULSIS. Am. J. Respir. Crit. Care Med 193:178-85 [PubMed: 26393389]

28. Kolb M, Richeldi L, Behr J, et al. 2017 Nintedanib in patients with idiopathic pulmonary fibrosis and preserved lung volume. Thorax 72(4):340-46 [PubMed: 27672117]

28a. Nathan SD, Meyer KC 2014 IPF Clinical trial design and endpoints. Curr Opin Pulm Med. 20:463-71. [PubMed: 25022315]

29. Nathan SD, Albera C, Bradford WZ, et al. 2017 Effect of pirfenidone on mortality: pooled analyses and meta-analyses of clinical trials in idiopathic pulmonary fibrosis. Lancet Respir. Med 5:33-41 [PubMed: 27876247]

30. Vancheri C, Kreuter M, Richeldi L, et al. 2018 Nintedanib with add-on pirfenidone in idiopathic pulmonary fibrosis. Results of the INJOURNEY trial. Am. J. Respir. Crit. Care Med 197:356-63 [PubMed: 28889759]

31. Raghu G, Rochwerg B, Zhang Y, et al. 2015 An official ATS/ERS/JRS/ALAT clinical practice guideline: treatment of idiopathic pulmonary fibrosis. An update of the 2011 clinical practice guideline. Am. J. Respir. Crit. Care Med 192:e3-19 [PubMed: 26177183]

32. Kreuter M, Wuyts W, Renzoni E, et al. 2016 Antacid therapy and disease outcomes in idiopathic pulmonary fibrosis: a pooled analysis. Lancet Respir. Med 4:381-89 [PubMed: 27050871]

33. Schaffer JM, Singh SK, Reitz BA, et al. 2015 Single- versus double-lung transplantation in patients with chronic obstructive pulmonary disease and idiopathic pulmonary fibrosis since the implementation of lung allocation based on medical need. JAMA 313:936-48 [PubMed: 25734735]

33a. Kondoh Y, Cottin V, Brown KK. 2017 Recent lessons learned in the management of acute exacerbation of idiopathic pulmonary fibrosis. Eur Resp Rev. 26:170050.

34. Patel NM, Lederer DJ, Borczuk AC, et al. 2007 Pulmonary hypertension in idiopathic pulmonary fibrosis. Chest 132:998-1006 [PubMed: 17873194] 
35. Gille T, Didier M, Boubaya M, et al. 2017 Obstructive sleep apnoea and related comorbidities in incident idiopathic pulmonary fibrosis. Eur. Respir. J 49(6):1601934 [PubMed: 28596432]

36. Lancaster LH, Mason WR, Parnell JA, et al. 2009 Obstructive sleep apnea is common in idiopathic pulmonary fibrosis. Chest 136:772-78 [PubMed: 19567497]

36a. Mermigkis C, Bouloukaki I, Schiza SE. 2017 Sleep as a new target for improving outcomes in idiopathic pulmonary fibrosis. Chest. 152:1327-38. [PubMed: 28774636]

37. Dowman LM, McDonald CF, Hill CJ, et al. 2017 The evidence of benefits of exercise training in interstitial lung disease: a randomised controlled trial. Thorax 72:610-19 [PubMed: 28213592]

38. Raghu G, Brown KK, Collard HR, et al. 2017 Efficacy of simtuzumab versus placebo in patients with idiopathic pulmonary fibrosis: a randomised, double-blind, controlled, phase 2 trial. Lancet Respir. Med 5:22-32 [PubMed: 27939076]

39. Raghu G, Martinez FJ, Brown KK, et al. 2015 CC-chemokine ligand 2 inhibition in idiopathic pulmonary fibrosis: a phase 2 trial of carlumab. Eur. Respir. J 46:1740-50 [PubMed: 26493793]

40. Parker JM, Glaspole IN, Lancaster LH, et al. 2018 A phase 2 randomized controlled study of tralokinumab in subjects with idiopathic pulmonary fibrosis. Am. J. Respir. Crit. Care Med 197:94-103 [PubMed: 28787186]

41. Mora AL, Rojas M, Pardo A, et al. 2017 Emerging therapies for idiopathic pulmonary fibrosis, a progressive age-related disease. Nat. Rev. Drug Discov 16:755-72 [PubMed: 28983101]

42. Oldham JM, Ma SF, Martinez FJ, et al. 2015 TOLLIP, MUC5B and the response to Nacetylcysteine among individuals with idiopathic pulmonary fibrosis. Am. J. Respir. Crit. Care Med 192(12):1475-82 [PubMed: 26331942]

43. Kropski JA, Blackwell TS, Loyd JE. 2015 The genetic basis of idiopathic pulmonary fibrosis. Eur. Respir. J 45(6):1717-27 [PubMed: 25837031]

44. Fingerlin TE, Murphy E, Zhang W, et al. 2013 Genome-wide association study identifies multiple susceptibility loci for pulmonary fibrosis. Nat. Genet 45:613-20 [PubMed: 23583980]

45. Allen RJ, Porte J, Braybrooke R, et al. 2017 Genetic variants associated with susceptibility to idiopathic pulmonary fibrosis in people of European ancestry: a genome-wide association study. Lancet Respir. Med 5:869-80 [PubMed: 29066090]

46. Noth I, Zhang Y, Ma SF, et al. 2013 Genetic variants associated with idiopathic pulmonary fibrosis susceptibility and mortality: a genome-wide association study. Lancet Respir. Med 1:309-17 [PubMed: 24429156]

47. Seibold MA, Wise AL, Speer MC, et al. 2011 A common MUC5B promoter polymorphism and pulmonary fibrosis. N. Engl. J. Med 364:1503-12 [PubMed: 21506741]

48. Helling BA, Gerber AN, Kadiyala V, et al. 2017 Regulation of MUC5B expression in idiopathic pulmonary fibrosis. Am. J. Respir. Cell Mol. Biol 57:91-99 [PubMed: 28272906]

49. Peljto AL, Zhang Y, Fingerlin TE, et al. 2013 Association between the MUC5B promoter polymorphism and survival in patients with idiopathic pulmonary fibrosis. JAMA 309:2232-39 [PubMed: 23695349]

49a. Peljto AL, Selman M, Kim DS, et al. 2015 The MUC5B promoter polymorphism is associated with idiopathic pulmonary fibrosis in a Mexican cohort but is rare among Asian ancestries. Chest. 147:460-64. [PubMed: 25275363]

50. Roy MG, Livraghi-Butrico A, Fletcher AA, et al. 2014 Muc5b is required for airway defence. Nature 505:412-16 [PubMed: 24317696]

51. Armanios MY, Chen JJ, Cogan JD, et al. 2007 Telomerase mutations in families with idiopathic pulmonary fibrosis. N. Engl. J. Med 356:1317-26 [PubMed: 17392301]

52. Tsakiri KD, Cronkhite JT, Kuan PJ, et al. 2007 Adult-onset pulmonary fibrosis caused by mutations in telomerase. PNAS 104:7552-57 [PubMed: 17460043]

53. Cogan JD, Kropski JA, Zhao M, et al. 2015 Rare variants in RTEL1 are associated with familial interstitial pneumonia. Am. J. Respir. Crit. Care Med 191(6):646-55 [PubMed: 25607374]

54. Kannengiesser C, Borie R, Menard C, et al. 2015 Heterozygous RTEL1 mutations are associated with familial pulmonary fibrosis. Eur. Respir. J 46:474-85 [PubMed: 26022962] 
55. Stuart BD, Choi J, Zaidi S, et al. 2015 Exome sequencing links mutations in PARN and RTEL1 with familial pulmonary fibrosis and telomere shortening. Nat. Genet 47:512-17 [PubMed: 25848748]

56. Deleted in proof [**AU: Ref 56 is a duplicate of Ref 54 and is not cited in text. We will renumber references and citations.**]

57. Kropski JA, Mitchell DB, Markin C, et al. 2014 A novel dyskerin (DKC1) mutation is associated with familial interstitial pneumonia. Chest 146:e1-7 [PubMed: 24504062]

58. Deleted in proof [**AU: Ref 58 is a duplicate of Ref 57 and is not cited in text. We will renumber references and citations.**]

59. Alder JK, Stanley SE, Wagner CL, et al. 2014 Exome sequencing identifies mutant TINF2 in a family with pulmonary fibrosis. Chest 147(5):1361-68

60. Kropski JA, Reiss S, Markin C, et al. 2017 Rare genetic variants in PARN are associated with pulmonary fibrosis in families. Am. J. Respir. Crit. Care Med 196:1481-84 [PubMed: 28414520]

60a. Stanley SE, Gable DL, Wagner CL. 2016 Loss-of-function mutations in the RNA biogenesis factor NAF1 predispose to pulmonary fibrosis-emphysema. Sci Transl Med. 8:351ra107.

61. Thomas AQ, Lane K, Phillips J, 3rd, et al. 2002 Heterozygosity for a surfactant protein C gene mutation associated with usual interstitial pneumonitis and cellular nonspecific interstitial pneumonitis in one kindred. Am. J. Respir. Crit. Care Med 165:1322-28 [PubMed: 11991887]

62. Fernandez BA, Fox G, Bhatia R, et al. 2012 A Newfoundland cohort of familial and sporadic idiopathic pulmonary fibrosis patients: clinical and genetic features. Respir. Res 13:64 [PubMed: 22853774]

63. van Moorsel CH, van Oosterhout MF, Barlo NP, et al. 2010 Surfactant protein C mutations are the basis of a significant portion of adult familial pulmonary fibrosis in a Dutch cohort. Am. J. Respir. Crit. Care Med 182:1419-25 [PubMed: 20656946]

64. Wang Y, Kuan PJ, Xing C, et al. 2009 Genetic defects in surfactant protein A2 are associated with pulmonary fibrosis and lung cancer. Am. J. Hum. Genet 84:52-59 [PubMed: 19100526]

65. Campo I, Zorzetto M, Mariani F, et al. 2014 A large kindred of pulmonary fibrosis associated with a novel ABCA3 gene variant. Respir. Res 15:43 [PubMed: 24730976]

66. Epaud R, Delestrain C, Louha M, et al. 2014 Combined pulmonary fibrosis and emphysema syndrome associated with ABCA3 mutations. Eur. Respir. J 43:638-41 [PubMed: 24136335]

67. Alder JK, Chen JJ, Lancaster L, et al. 2008 Short telomeres are a risk factor for idiopathic pulmonary fibrosis. PNAS 105:13051-56 [PubMed: 18753630]

68. Diaz de Leon A, Cronkhite JT, Katzenstein AL, et al. 2010 Telomere lengths, pulmonary fibrosis and telomerase (TERT) mutations. PLOS ONE 5:e10680 [PubMed: 20502709]

69. Lawson WE, Grant SW, Ambrosini V, et al. 2004 Genetic mutations in surfactant protein C are a rare cause of sporadic cases of IPF. Thorax 59:977-80 [PubMed: 15516475]

70. Petrovski S, Todd JL, Durheim MT, et al. 2017 An exome sequencing study to assess the role of rare genetic variation in pulmonary fibrosis. Am. J. Respir. Crit. Care Med 196(1):82-93 [PubMed: 28099038]

71. Dressen A, Abbas A, Cabanski C, et al. 2018 Functional variants of telomerase genes are enriched in IPF patients lacking the MUC5B common risk allele. Lancet Respir. Med[E-published]

72. Kropski JA, Young LR, Cogan JD, et al. 2017 Genetic evaluation and testing of patients and families with idiopathic pulmonary fibrosis. Am. J. Respir. Crit. Care Med 195(11):1423-28 [PubMed: 27786550]

73. Lawson WE, Crossno PF, Polosukhin VV, et al. 2008 Endoplasmic reticulum stress in alveolar epithelial cells is prominent in IPF: association with altered surfactant protein processing and herpesvirus infection. Am. J. Physiol. Lung Cell Mol. Physiol 294:L1119-26 [PubMed: 18390830]

74. Lawson WE, Cheng DS, Degryse AL, et al. 2011 Endoplasmic reticulum stress enhances fibrotic remodeling in the lungs. PNAS 108:10562-67 [PubMed: 21670280]

75. Korfei M, Ruppert C, Mahavadi P, et al. 2008 Epithelial endoplasmic reticulum stress and apoptosis in sporadic idiopathic pulmonary fibrosis. Am. J. Respir. Crit. Care Med 178:838-46 [PubMed: 18635891] 
76. Stuart BD, Lee JS, Kozlitina J, et al. 2014 Effect of telomere length on survival in patients with idiopathic pulmonary fibrosis: an observational cohort study with independent validation. Lancet Respir. Med 2:557-65 [PubMed: 24948432]

77. Kropski JA, Pritchett JM, Zoz DF, et al. 2015 Extensive phenotyping of individuals at risk for familial interstitial pneumonia reveals clues to the pathogenesis of interstitial lung disease. Am. J. Respir. Crit. Care Med 191:417-26 [PubMed: 25389906]

78. Deleted in proof [**AU: Ref 78 is a duplicate of Ref 77 and is not cited in text. We will renumber references and citations.**]

79. Snetselaar R, van Batenburg AA, van Oosterhout MFM, et al. 2017 Short telomere length in IPF lung associates with fibrotic lesions and predicts survival. PLOS ONE 12:e0189467 [PubMed: 29281671]

80. Naikawadi RP, Disayabutr S, Mallavia B, et al. 2016 Telomere dysfunction in alveolar epithelial cells causes lung remodeling and fibrosis. JCI Insight 1:e86704 [PubMed: 27699234]

81. Povedano JM, Martinez P, Flores JM, et al. 2015 Mice with pulmonary fibrosis driven by telomere dysfunction. Cell Rep. 12:286-99 [PubMed: 26146081]

82. Bueno M, Lai YC, Romero Y, et al. 2015 PINK1 deficiency impairs mitochondrial homeostasis and promotes lung fibrosis. J. Clin. Investig 125:521-38 [PubMed: 25562319]

83. Bueno M, Brands J, Voltz L, et al. 2018 ATF3 represses PINK1 gene transcription in lung epithelial cells to control mitochondrial homeostasis. Aging Cell 17(2):e12720

84. Yu G, Tzouvelekis A, Wang R, et al. 2018 Thyroid hormone inhibits lung fibrosis in mice by improving epithelial mitochondrial function. Nat. Med 24:39-49 [PubMed: 29200204]

85. Nabhan AN, Brownfield DG, Harbury PB, et al. 2018 Single-cell Wnt signaling niches maintain stemness of alveolar type 2 cells. Science 359:1118-23 [PubMed: 29420258]

86. Zacharias WJ, Frank DB, Zepp JA, et al. 2018 Regeneration of the lung alveolus by an evolutionarily conserved epithelial progenitor. Nature 555:251-55 [PubMed: 29489752]

87. Liang J, Zhang Y, Xie T, et al. 2016 Hyaluronan and TLR4 promote surfactant-protein-C-positive alveolar progenitor cell renewal and prevent severe pulmonary fibrosis in mice. Nat. Med 22:1285-93 [PubMed: 27694932]

88. Xie T, Liang J, Liu N, et al. 2016 Transcription factor TBX4 regulates myofibroblast accumulation and lung fibrosis. J. Clin. Investig 126:3063-79 [PubMed: 27400124]

89. Herrera J, Henke CA, Bitterman PB. 2018 Extracellular matrix as a driver of progressive fibrosis. J. Clin. Investig 128:45-53 [PubMed: 29293088]

90. Tschumperlin DJ, Ligresti G, Hilscher MB, et al. 2018 Mechanosensing and fibrosis. J. Clin. Investig. 128:74-84 [PubMed: 29293092]

91. Larson-Casey JL, Deshane JS, Ryan AJ, et al. 2016 Macrophage Akt1 kinase-mediated mitophagy modulates apoptosis resistance and pulmonary fibrosis. Immunity 44:582-96 [PubMed: 26921108]

92. Young LR, Gulleman PM, Short CW, et al. 2016 Epithelial-macrophage interactions determine pulmonary fibrosis susceptibility in Hermansky-Pudlak syndrome. JCI Insight 1:e88947 [PubMed: 27777976]

93. Satoh T, Nakagawa K, Sugihara F, et al. 2017 Identification of an atypical monocyte and committed progenitor involved in fibrosis. Nature 541:96-101 [PubMed: 28002407]

94. Xi Y, Kim T, Brumwell AN, et al. 2017 Local lung hypoxia determines epithelial fate decisions during alveolar regeneration. Nat. Cell Biol 19:904-14 [PubMed: 28737769]

95. Xu Y, Mizuno T, Sridharan A, et al. 2016 Single-cell RNA sequencing identifies diverse roles of epithelial cells in idiopathic pulmonary fibrosis. JCI Insight 1:e90558 [PubMed: 27942595]

96. Xie T, Wang Y, Deng N, et al. 2018 Single-cell deconvolution of fibroblast heterogeneity in mouse pulmonary fibrosis. Cell Rep. 22:3625-40 [PubMed: 29590628] 


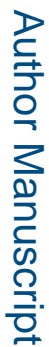

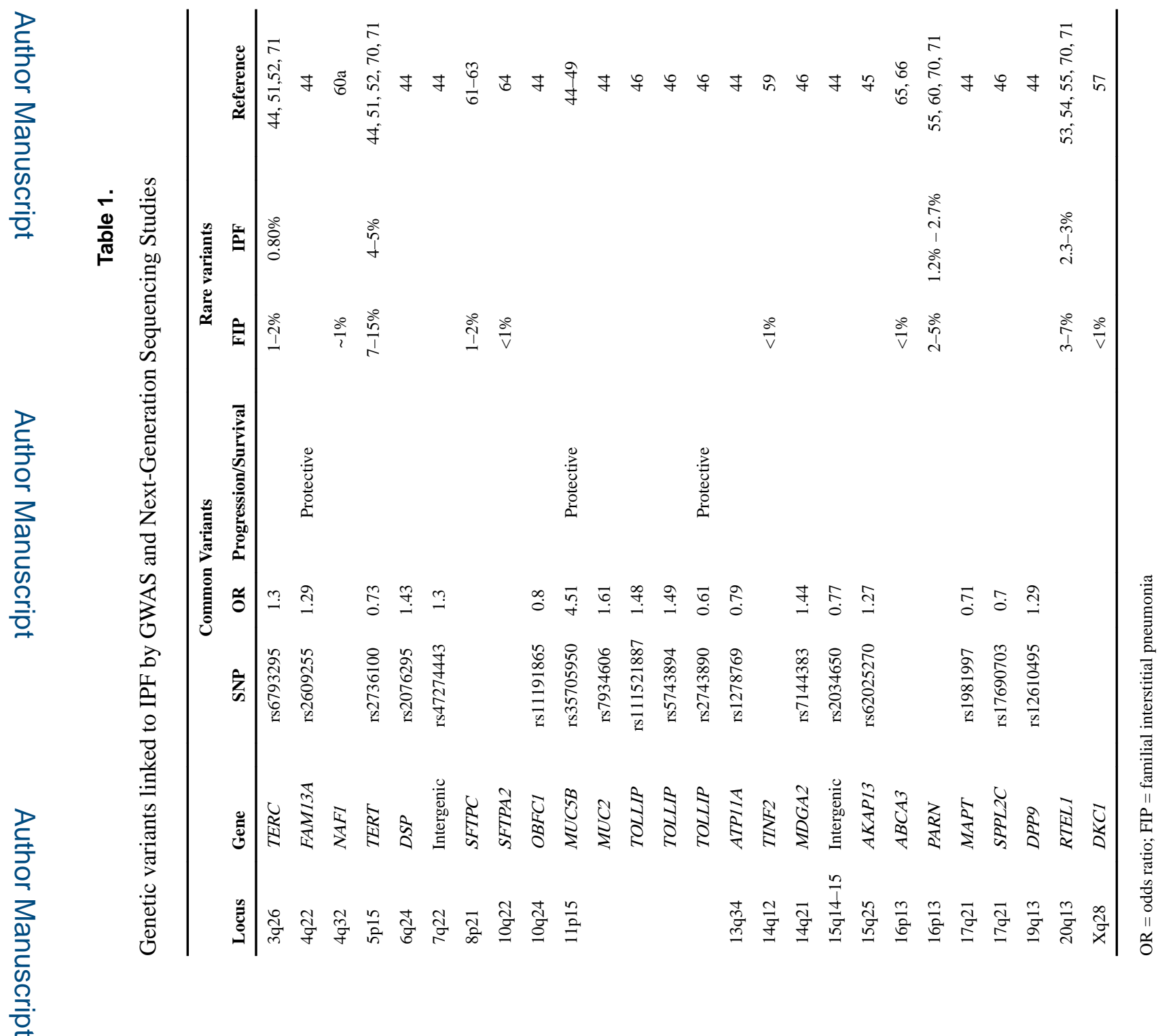

